\title{
Finger Vein Recognition using Rotated Wavelet Filters
}

\author{
Santosh P. Shrikhande \\ School of Technology, \\ Swami Ramanand Teerth \\ Marathwada University, \\ Sub-Centre, Latur, Maharashtra, India
}

\author{
H. S. Fadewar \\ School of Computational Sciences, \\ Swami Ramanand Teerth \\ Marathwada University, \\ Nanded, Maharashtra, India
}

\begin{abstract}
Finger vein biometric have been recognized as the most effective and promising recognition method due to its accuracy and security. This paper discusses a method for finger vein image features extraction using 2-D Rotated Wavelet Filters (RWF) and Discrete Wavelet Transform (DWT) jointly. A set of 2-D RWF filters improves characterization of diagonally oriented texture features from a finger vein image. The 2-D RWF and DWT jointly used for decomposition of a finger vein image ROI up to third level. The standard deviation and energy of each subband from every decomposition level are used for the creation of features vector. Then Canberra distance classifier is used for the classification of finger vein images. The performance of this method has evaluated on the standard finger vein image database of Shandong University (SDUMLA), China. Experimental results have shown that the method with RWF and DWT jointly gives better results as compare to the traditional DWT based methods.
\end{abstract}

\section{General Terms}

Pattern recognition and classification, Biometrics, Wavelet Transform.

\section{Keywords}

Finger Vein Recognition, Discrete Wavelet Transform, Rotated Wavelet Filters.

\section{INTRODUCTION}

Nowadays, personal identification and verification using biometric technology has been used in many applications such as door access control, ATM transactions and border crossing controls [1]. Biometrics is an automated method of identifying and verifying the persons based on their physiological or behavioral characteristics such as finger print, palm print, face, iris, retina, voice, gait and signatures [2]. Finger print is the most popular and mature biometric but it is vulnerable to forgery because the fingerprints are easily exposed to the others. In addition, the condition of the finger surfaces such as sweat and dryness can degrade the performance and accuracy $[1,2]$. To overcome the limitations of finger print biometric system, finger vein biometric has been mostly used. Finger vein biometric is the technology of identifying and verifying the people using their finger veins pattern based on the fact that every individual has unique vein pattern [3]. The human body veins circulate the blood towards heart. The veins pattern cannot be seen by necked eyes and normal camera. The camera with infrared light of $740-960 \mathrm{~nm}$ wavelength penetrates the user's finger as it is held over the sensor. The hemoglobin in blood veins absorbs the infrared light and reflects veins image as black dark lines and other part as a bright $[2,3,4]$. Finger vein biometric has several benefits as compared to other hand based biometrics. First, the finger vein pattern is hard to replicate and forge since it is an internal feature. Second, the quality of the captured vein pattern image is not influenced by skin condition such as sweat, dryness and

skin diseases. Lastly, finger vein image capturing is hygienic because it does not require contact between the finger and sensor [5, 6]. Due to above benefits finger vein recognition has become popular, promising and efficient biometric for personal identification $[2,5]$.

\subsection{Related Work}

The different methods from literature to extract the local and global vein pattern features from the finger vein images are discussed below:

In [3], Miura et al. used repeated line tracking algorithm to extracts vein pattern features from the finger vein image. The line-tracking operation starts randomly at any pixel and move pixel by pixel along the dark line. Experimental results have shown $0.145 \%$ as equal error rate (EER) and $460 \mathrm{~ms}$ response time.

In [4], Miura et al. proposed a method to extract the local maximum curvature points from the cross sectional profiles of a finger vein image for recognition. A data set of 678 different finger vein images with two samples of each was used for conducting an experiment. Experimental results have shown the equal error rate as $0.0009 \%$.

In [6], Cheng-Bo et al. proposed a method to extract the vein pattern features with minutiae features such as bifurcation points and ending points from the finger vein images. These feature points were used as a geometric representation of the vein pattern shape. Then Modified Hausdorff Distance (MHD) algorithm was proposed for matching of relative positions of the shape of vein. A data set of 50 persons with 10 samples of each was used for conducting an experiment. Experimental results have shown the equal error rate $0.761 \%$ where the HD distances threshold value was 0.43 .

In [7], Liu et al. presented a modified repeated line tracking algorithm to extract width of the finger vein and parameters were revised according to the vein width for line tracking to figure out locus space of finger vein. Finally, the processing results were obtained using Otsu's method for exact segmentation on the locus space. Experiments have shown that the proposed method is more robust and efficient than traditional repeated line tracking algorithm.

Local features using minutiae points, maximum curvatures and repeated line tracking methods are found very difficult to segment and extract the minutiae points and curvatures from poor quality finger vein images. To overcome this limitation of minutiae based method, Local Binary Pattern (LBP) based methods were researched to extract LBP codes [8].

In [10], Rosdi et al. used Local Line Binary Pattern (LLBP) to overcome the problem of LBP. The shape of the 
neighborhood was line in LLBP unlike square in LBP. The magnitude of LLBP was obtained by calculating the line binary codes for both horizontal and vertical components. Experimental results have shown that the LLBP method has significantly lower equal error rate than the LBP based methods.

LBP and LLBP based methods requires more memory space to store a feature matrix of LBP code of an input image. To overcome the limitations of minutiae and LBP based methods, Yang et al. [11], used a bank of even symmetric Gabor filters to exploit the local and global finger vein features from FVCodes in multi-scale and multi-orientation. The finger vein classification was done using the cosine similarity classifier and a fusion scheme in decision level. The performance of the Gabor based method were found good in finger vein recognition.

\subsection{Motivation}

Most of the finger vein features extraction methods from literature were used local vein structure and local binary pattern for feature extraction. But these methods were found not better in poor quality finger vein images. The performance of Gabor based features extraction method with poor quality images were found better than local veins structure but with high computational complexity and more storage space. So to overcome this limitation, standard DWT based methods were used in [15, 16, 18 and 19] to extract the global texture features from finger print and face images. Standard wavelet gives the horizontal, vertical and diagonal features oriented along the angles of $\left\{0^{\circ}, 90^{\circ}\right.$ and $\left.\pm 45^{\circ}\right\}$ but does not provide an information strongly oriented at angles of $\left\{45^{0}, 135^{\circ}\right\}$ in the separate subbands. To overcome this limitation of standard wavelet, Kokare et al. [12] designed 2D Rotated Wavelet Filters (RWF) and jointly used with standard DWT to extract significant directional features from texture with less computational complexity and less storage space. Hence we motivated to use these 2D RWF filters combined with standard DWT to extract significant directional features oriented at $\left\{45^{0}, 135^{\circ}\right\}$ from the finger vein image which cannot be distinguished by the standard DWT.

Rest of the paper is organized as follows. In Section 2, we briefly discuss DWT. Rotated Wavelet Filters are discussed in Section 3. The proposed finger vein recognition method is given in Section 4. Experimental results and discussions are given in Section 5. Finally the conclusion is provided in the Section 6 .

\section{DISCRETE WAVELET TRANSFORM}

Discrete Wavelet Transform (DWT) is a popular mathematical tool used for transforming and analyzing the signal in the time and frequency domain [13, 18]. The standard DWT decomposes an image $\mathrm{f}(\mathrm{x}, \mathrm{y})$ in terms of the shifted and dilated wavelet functions at $\left\{\Psi 0^{0}, \Psi 90^{\circ}, \Psi \pm 45^{\circ}\right\}$ and the scaling function $\phi(\mathrm{x}, \mathrm{y})$ are represented by equation (1) and (2) respectively [13].

$$
W_{\phi}\left(j_{0}, k_{1}, k_{2}\right)=\frac{1}{\sqrt{M N}} \sum_{x=0}^{M-1} \sum_{y=0}^{N-1} f(x, y) \phi_{0, k_{1}, k_{2}}(x, y)
$$

$$
W_{\psi}^{i}\left(j_{0}, k_{1}, k_{2}\right)=\frac{1}{\sqrt{M N}} \sum_{x=0}^{M-1} \sum_{y=0}^{N-1} f(x, y) \psi_{j_{0}, k_{1}, k_{2}}^{i}(x, y)
$$

Where $\mathrm{i}=\theta$ i.e. angle $\left\{0^{\circ}, 90^{\circ}\right.$ and $\left.\pm 45^{\circ}\right\}$ of wavelet function. The $2 \mathrm{D}$ scaling and wavelet functions can be realized using separable 1D wavelet filters by applying them along the rows and columns one after the other at each level of decomposition. The standard DWT decomposes an input image into the four subbands such as LL, LH, HL and HH. The LL subband (Low frequency components row and column wise) gives an approximate coefficients i.e. an average image. The LH subband (Low frequency components row and High frequency component column wise), the HL subband (High frequency components row and Low frequency component column wise), and the $\mathrm{HH}$ subband (High frequency components row and column wise) gives horizontal, vertical and diagonal information of an input image respectively $[13,15]$.

\section{ROTATED WAVELET FILTERS (RWF)}

The 2D non separable wavelet filters are designed using 1D separable wavelet filter coefficients by taking the product of scaling and wavelet function [12]. The high pass and low pass 1D Daubechies eight tap wavelet filter coefficients are extracted by using the wfilters ( ) function in MATLAB. The $2 \mathrm{D}$ wavelet filters $[12,13]$ are created using $1 \mathrm{D}$ wavelet filter coefficients using equation (3).

$$
\begin{aligned}
& I_{l l}=\phi(i) \phi(j) \\
& I_{l h}=\phi(i) \psi(j) \\
& I_{h l}=\psi(i) \phi(j) \\
& I_{h h}=\psi(i) \psi(j)
\end{aligned}
$$

where $\phi$ and $\Psi$ are the scaling and wavelet functions respectively.

The 2D wavelet filters $\mathrm{H}_{\mathrm{ll}}, \mathrm{H}_{\mathrm{lh}}, \mathrm{H}_{\mathrm{hl}}$ and $\mathrm{H}_{\mathrm{hh}}$ are designed from Daubechies eight tap wavelet low pass (h) and high pass (g) filter coefficients using the matrix operations [12] by equation (4).

$$
\begin{aligned}
& T I_{I I}=h^{T}=1=h \\
& I_{\text {In }}=h^{T}=5 \\
& T I_{h l}=8^{T=1 h} \\
& T_{n h}=5^{T=1}
\end{aligned}
$$

The 2D Rotated Wavelet Filters (RWF) such as $\mathrm{HR}_{\mathrm{ll}}, \mathrm{HR}_{\mathrm{lh}}$, $\mathrm{HR}_{\mathrm{hl}}$ and $\mathrm{HR}_{\mathrm{hh}}$ are constructed by rotating the $2 \mathrm{D}$ wavelet filters by $45^{\circ}$ angles in MATLAB using imrotate function. If $\mathrm{N}$ is the length of $1 \mathrm{D}$-wavelet filter, then $(2 \mathrm{~N}-1) \times(2 \mathrm{~N}-1)$ is the length of 2D RWF. The given input image $f(x, y)$ is decomposed by filtering with $2 \mathrm{D}$ RWF filters followed by the 2D down sampling at each level $[12,13]$. These constructed RWF filters extracts diagonally oriented information $\left(45^{0}\right)$ into $\mathrm{LH}$ and $\left(135^{\circ}\right)$ into HL subbands respectively, which cannot be distinguished by the standard DWT $[12,13]$. The Fig. 1 shows the four subbands $H_{\mathrm{ll}}, \mathrm{HR}_{\mathrm{lh}}, \mathrm{HR}_{\mathrm{hl}}$ and $\mathrm{HR}_{\mathrm{hh}}$ resulted from second level decomposition using RWF filters [12]. 


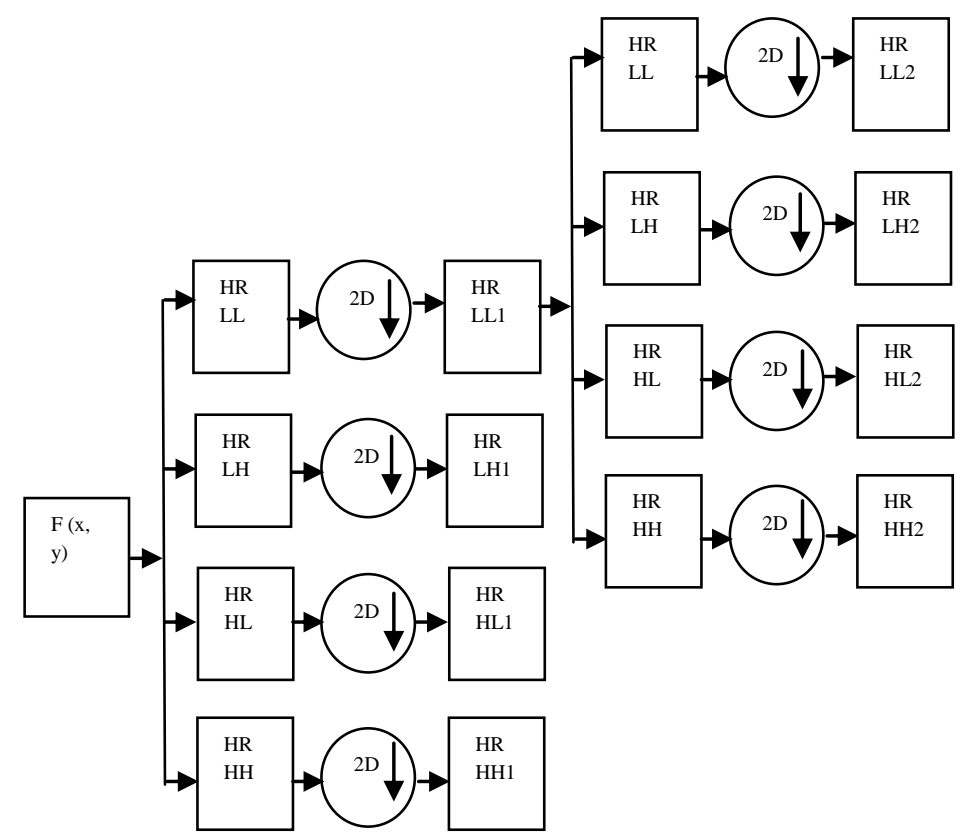

Fig 1: Second level decomposition using 2D-RWF filters

\section{FINGER VEIN FEATURES EXTRACTION AND MATCHING}

In this research work, the features of finger vein image are extracted using standard DWT, RWF and the combination of DWT and RWF.

\subsection{Finger Vein Image Database}

In this research work, the finger vein image database is used which has provided by the Machine Learning and Data Mining Lab, Shandong University (SDUMLA), China [14]. These finger vein images have been captured by a device which was designed by Joint Lab for Intelligent Computing and Intelligent Systems of Wuhan University. The finger vein images in this database were collected from 106 volunteers consisting with index, middle and ring finger of both left and right hand of each. The total finger veins images available are equal to 106 subjects with 6 fingers in 5 variations i.e. (106 x $6 \times 5=3180$ ) images. The forefinger and middle finger of both left and right hands of 34 individuals with 20 variations of each $(34 \times 4 \times 20=2720)$ finger vein image database were provided by the [9] used for conducting an experiment.

\subsection{Preprocessing}

The original images from the existing database were 24-bit color images with a size of $320 \times 240$ pixels. These original images are transformed into 8-bit gray scale image and Sobel edge detection operator is used for detecting the edges of finger from a finger vein image. Then the width and height of finger image is calculated using the maximum and minimum abscissa values of the finger profile [9]. Then the ROI of finger vein image is extracted based on these abscissa values and its size is normalized to $96 \times 64$ pixels using the bilinear interpolation [9].

\subsection{Features Extraction}

The 96x64 pixels ROI of a finger vein image is decomposed by applying the 2D standard DWT, 2D RWF and 2D DWT + RWF jointly up to third level decomposition. Because beyond the third level decomposition, it does not increase the recognition rate but increases the computational complexity [13]. The statistical features such as standard deviation (Sk) and absolute mean i.e. energy (Ek) from a decomposed image are extracted using equation (5) and (6) respectively.

$$
\begin{gathered}
S_{k}=\sqrt{\frac{1}{M N} \sum_{i=1}^{M} \sum_{j=1}^{N}\left(S b_{i j}-\mu_{k}\right) 2} \\
E_{k}=\frac{1}{M N} \sum_{i=1}^{M} \sum_{j=1}^{N}|S b i j|
\end{gathered}
$$

where Sk and Ek are the standard deviation and energy of the $\mathrm{k}^{\text {th }}$ subband respectively. $\mathrm{M}, \mathrm{N}$ is the size and $\mu \mathrm{k}$ is the mean of $\mathrm{k}^{\text {th }}$ decomposed subband $(\mathrm{Sb})$. The standard deviation and energy of each subband is used for the creation of feature vector. The length of feature vector using different feature set is equal to the (No. of features $\times$ No. of decomposed subbands). Feature vectors of forefinger and middle finger of left and right hand is used for enrollment purpose. The feature vector length using DWT features set is equal to the (12 subbands $* 2$ features $=24$ elements). Where the features are two i.e. standard deviation (Sk) and energy (Ek). The feature vector length using DWT+RWF feature set will be double of DWT. These feature vectors subject wise are arranged in a matrix form into the feature database. Four different feature sets are created using DWT, RWF and DWT+RWF jointly. The feature Set 1 is constructed using only DWT features. Feature Set 2 is created using only RWF. The feature Set 3 is created by using combination of DWT and RWF. Lastly the feature Set 4 is formed using DWT and RWF (only LH \& HL) subbands jointly. Then the analysis is performed by decomposing an input finger vein image up to third level.

\subsection{Finger Vein Matching}

A query or test image is an image which user wants to test or verify his identity from the feature database. The feature vector of query image is computed and compared with the feature database. Feature vectors of all the variation of forefinger and middle finger of each individual's left and right hand is used for the testing purpose. The proposed method has used Canberra distance classifier based classification of an image. The distances in between query image and all enrolled images are computed and stored into the Distance matrix 
subject wise. A row index where the minimum distance found is computed from this distance matrix. This computed row index is the nearest matched subject with the query image. If $\mathrm{X}$ and $\mathrm{Y}$ are the feature vectors of the query image and enrolled image respectively, then the Canberra distance $(\mathrm{Cn})$ in between $\mathrm{X}$ and $\mathrm{Y}$ feature vectors is given below:

$$
C n=\sum_{i=1}^{n} \frac{\left|X_{i}-Y_{i}\right|}{\left|X_{i}\right|+\left|Y_{i}\right|}
$$

Canberra distance classifier method is considered to be a good expression to use, which avoids the scaling effect. Because, in above equation (7), the numerator signifies the difference and denominator normalizes the difference. Hence the distance value will never exceeds one and it is equal to one only when either of the attribute is zero [12, 13 and 15].

The steps of the proposed method for the finger vein features extraction and recognition process are given below:

Step 1: Input finger vein image ROI is extracted and size is normalized to $96 \times 64$ pixels.

Step 2: Non separable 2D Wavelet filters are designed in MATLAB as follows;

(a) 1D Daubechies eight tap wavelet filter coefficients are taken in MATLAB using wfilters () function. Low pass filter coefficients are taken in ' $h$ ' and high pass filter coefficients in ' $\mathrm{g}$ '.

(b) 2D wavelet filters are constructed by taking the product i.e. matrix operations[12] such as;

$$
\begin{array}{ll}
\mathrm{H}_{\mathrm{ll}}=\mathrm{h}^{\mathrm{T}} \mathrm{h} ; & \mathrm{H}_{\mathrm{lh}}=\mathrm{h}^{\mathrm{T}} \mathrm{g} ; \\
\mathrm{H}_{\mathrm{hl}}=\mathrm{g}^{\mathrm{T}} \mathrm{h} ; & \mathrm{H}_{\mathrm{hh}}=\mathrm{g}^{\mathrm{T}} \mathrm{g} ;
\end{array}
$$

Step 3: 2D Rotated Wavelet Filters are constructed in MATLAB;

(a) The 2D RWF are constructed [12] by rotating the 2D wavelet filters $\mathrm{H}_{\mathrm{ll}}, \mathrm{H}_{\mathrm{lh}}, \mathrm{H}_{\mathrm{hl}}$ and $\mathrm{H}_{\mathrm{hh}}$ individually with $45^{0}$ degree using imrotate ( ) function in MATLAB

Step 4: These constructed 2D RWF filters are convolved with ROI of finger vein image and fallowed by the $2 \mathrm{D}$ down sampling up to the third level decomposition.

Step 5: The statistical features such as standard deviation (Sk) and absolute mean i.e. energy (Ek) are extracted from each subband of every decomposition level. These features elements into the feature vectors are enrolled as the template i.e. feature database.

Step 6: The Canberra distances in between the query image and all enrolled images subject wise are calculated and arranged in a matrix form called as a Distance Matrix.

Step 7: The row index is searched where the minimum Canberra distance is found in the Distance Matrix. This row number is the subject number to which the query image is nearly matches.

\section{EXPERIMENTAL RESULTS AND DISSCUSION}

The finger vein image database provided by Shandong University (SDUMLA), China [14] has been used for conducting the experiments. Experiments are conducted on
Core i3 machine with $2.40 \mathrm{GHz}$ processor and 2GB RAM using MATLAB 2013a. The GUI application is developed using MATLAB for feature extraction using four feature sets one at a time, then these feature vectors are enrolled into the feature database. Then the Canberra distances in between query and enrolled images are calculated and stored into the Distance Matrix. Minimum distance row is the subject number to which query image matches. The recognition results of the proposed method are compared with the standard DWT based method. The comparison of DWT, RWF and DWT+RWF jointly with energy and standard deviation features has shown in the Table 1.

Table 1. Recognition Rates of Proposed and DWT Method

\begin{tabular}{|c|c|c|c|}
\hline \multirow{2}{*}{ Method } & Features & \multicolumn{2}{|c|}{ Recognition Rates } \\
\cline { 3 - 4 } & GAR & FRR \\
\hline $\begin{array}{c}\text { DWT + } \\
\text { RWF ( LH } \\
\text { and HL } \\
\text { Subbands ) }\end{array}$ & $\begin{array}{c}\text { Standard } \\
\text { deviation + } \\
\text { Energy }\end{array}$ & 93.67 & 6.33 \\
\hline $\begin{array}{c}\text { DWT + RWF } \\
\text { (All } \\
\text { Subbands) }\end{array}$ & $\begin{array}{c}\text { Standard } \\
\text { deviation + } \\
\text { Energy }\end{array}$ & 92.86 & 7.14 \\
\hline $\begin{array}{c}\text { Only DWT } \\
\text { Ondandard } \\
\text { Energy }\end{array}$ & 91.10 & 8.90 \\
\hline Only RWF & $\begin{array}{c}\text { Standard } \\
\text { deviation + } \\
\text { Energy }\end{array}$ & 63.68 & 36.32 \\
\hline
\end{tabular}

From the Table 1., It is clear that the proposed method with DWT + RWF (only LH and HL Subbands) feature set has given a $93.67 \%$ Genuine Acceptance Rate (GAR) at $6.33 \%$ False Rejection Rate (FRR) which outperforms all the other DWT+RWF and DWT based methods. The redundancy of the complete DWT + RWF feature set is reduced by excluding the $\mathrm{LL}$ and $\mathrm{HH}$ subbands at each level from features extraction. Because most of information is redundant due to the filter responses of $\mathrm{H}_{\mathrm{LL}}$ and rotated $\mathrm{H}_{\mathrm{LL}}$ and the filter response of $\mathrm{H}_{\mathrm{HH}}$ is totally covered by the filter response of the $\mathrm{H}_{\mathrm{LH}}$ and $\mathrm{H}_{\mathrm{LH}}$. Hence, almost diagonal oriented information from finger vein image is extracted from the $\mathrm{H}_{\mathrm{LH}}$ and $\mathrm{H}_{\mathrm{HL}}$ subbands of rotated wavelet filter responses. The features set using DWT+ RWF features has given a $92.86 \%$ GAR at $7.14 \%$ FRR which outperforms the traditional DWT based methods. Because RWF feature set has given some additional information i.e. diagonally oriented texture features to the DWT. Due to this, the recognition results of DWT+ RWF are better compare to the traditional DWT based methods.

The comparison of recognition rates of the DWT + RWF (only LH and HL), DWT + RWF and DWT based methods are shown in the Fig. 2. 


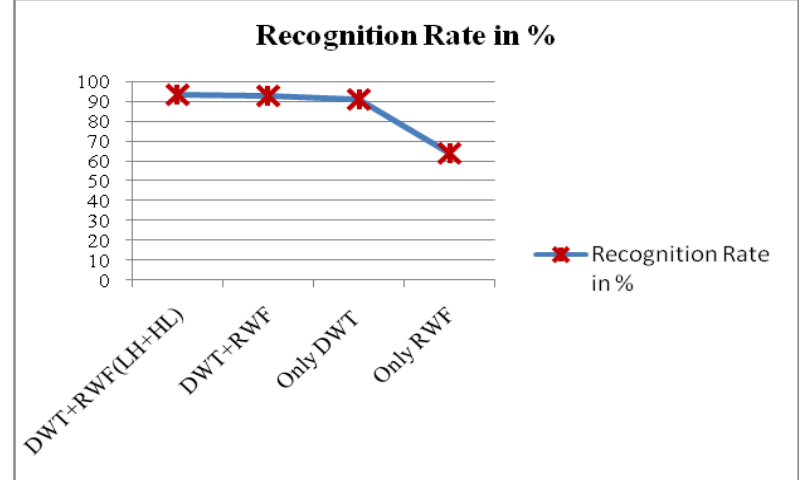

Fig 2: Comparison of Recognition Rates of Proposed and DWT Methods

The performance comparison of DWT + RWF (only LH and HL subband), DWT+RWF, RWF and DWT methods in terms of the feature vector length, average feature extraction time and average feature matching time is given in the Table 2 .

Table 2. Performance Measure of Proposed and DWT Methods

\begin{tabular}{|c|c|c|c|c|}
\hline $\begin{array}{c}\text { Feature } \\
\text { Measure }\end{array}$ & $\begin{array}{c}\text { DWT+R } \\
\text { WF (only } \\
\text { LH, HL ) }\end{array}$ & $\begin{array}{c}\text { DWT + } \\
\text { RWF }\end{array}$ & RWF & DWT \\
\hline $\begin{array}{c}\text { Feature } \\
\text { Vector } \\
\text { Length in } \\
\text { elements }\end{array}$ & 36 & 48 & 24 & 24 \\
\hline $\begin{array}{c}\text { Feature } \\
\text { extraction } \\
\text { time in } \\
\text { seconds }\end{array}$ & 0.1594 & 0.1692 & 0.1560 & 0.1525 \\
\hline $\begin{array}{c}\text { Matching } \\
\text { Time in } \\
\text { seconds }\end{array}$ & 0.0584 & 0.0698 & 0.0485 & 0.0418 \\
\hline
\end{tabular}

The above table shows the average feature extraction and matching time of individual method. The number of features i.e. features elements in the feature vector of proposed method with DWT+RWF (only LH and HL) are less as compare to the (DWT+RWF all subbands) feature set method. Hence, the time required to extract the features from finger vein image and compare the features for recognition is less for DWT + RWF (only LH, HL) method as compare to the other feature sets method.

\section{CONCLUSION AND FUTURE WORK}

In this paper, 2D Rotated Wavelet Filters designed by [12] and DWT has been used for the finger vein feature extraction and Canberra distance classifier for classification. The 2D non separable RWF filters are constructed using 1D separable wavelet filters and rotated them with $45^{\circ}$. These 2D RWF filters are applied on ROI of finger vein images to extract diagonally oriented features from $\left(45^{0}\right)$ and $\left(135^{0}\right)$ in a separate subband which cannot be extracted by the standard DWT. Experimental results have shown that the proposed method with DWT + (LH, HL subbands of RWF) has given better recognition results as compare to traditional DWT and with reduced feature vector dimensionality over DWT+RWF method. The recognition results of the DWT+RWF method outperforms the traditional DWT method due the complementary information provided by RWF to DWT. The future work is to improve the recognition accuracy by combining the local features of finger vein pattern using its structure and combine it with global features using DWT and RWF filters.

\section{ACKNOWLEDGMENTS}

The authors would like to thank Machine Learning and Data Mining Lab, Shandong University (SDUMLA), China for providing the finger vein image database to conduct an experiment. Authors also would like to thank all the anonymous reviewers, colleagues and friends for their suggestions and remarks for completion of this paper.

\section{REFERENCES}

[1] Kang Ryoung Park, "Finger Vein Recognition by Combining Global and Local Features on SVM", Computing and Informatics, Vol. 30, pp. 295-309, 2011

[2] Santosh P. Shrikhande and H. S. Fadewar, "Finger Vein Recognition Using Discrete Wavelet Packet Transform Based Features", International Conference on Advances in Computing, Communications and Informatics (ICACCI), Vol. 1, pp. 1646-1651, 2015

[3] Naoto Miura, Akio Nagasaka, Takafumi Miyatake, "Feature extraction of finger-vein patterns based on repeated line tracking and its application to personal identification", Machine Vision and Applications, 15: pp. 194-203, 2004

[4] Naoto Miura, Akio Nagasaka, "Extraction of FingerVein Patterns Using Maximum Curvature Points in Image Profiles", IAPR Conference on Machine Vision Applications, Tsukuba Science City, Japan, pp. 347-50, May 2005

[5] Jinfeng Yang, Yihua Shi, Jinli Yang, "Personal identification based on finger-vein feature", Computers in Human Behavior 27 (Elsevier) pp. 1565-1570, November 2010

[6] Cheng Bo Yu, Hua Feng Qin, Lian Zhang, Yan-Zhe Cui, "Finger vein image recognition combining modified hausdorff distance with minutiae feature matching", J. Biomedical Science and Engineering, pp. 261-272, August 2009

[7] Liu, J B Xie, W Yan, P Q Li and HZ Lu, "An algorithm for finger-vein segmentation based on modified repeated line tracking”, The Imaging Science Journal Vol 61, pp. 491-502

[8] Eui Chul Lee, Hyunwoo Jung and Daeyeoul Kim, "New Finger Biometric Method Using Near Infrared Imaging", Sensors, pp. 2319-2333, February 2011

[9] Xianjing Meng, Gongping Yang, Yilong Yin and Rongyang Xiao, " Finger Vein Recognition Based on Local Directional Code", Sensors, pp. 14937-14952, November 2012

[10] Bakhtiar Affendi Rosdi, Chai Wuh Shing and Shahrel Azmin Suandi, "Finger Vein Recognition Using Local Line Binary Pattern", Sensors 11, pp. 11357-11371, November 2011

[11] Jinfeng Yang, Yihua Shi and Renbiao Wu, "Finger-Vein Recognition Based on Gabor Features", Biometric Systems, Design and Applications, Published by Intech, pp. 17-32, 2011

[12] Manesh Kokare, P.K. Biswas, B.N. Chatterji, "Texture image retrieval using rotated wavelet filters", Pattern Recognition Letters 28, pp. 1240-1249, February 2007 
[13] Sangita Bharkad, Manesh Kokare, "Rotated Wavelet Filters-Based Fingerprint Recognition", International Journal of Pattern Recognition and Artificial Intelligence, Vol. 26, No. 3, pp. 1256008-1- 21, Septmber 2012

[14] Yilong Yin, Lili Liu, and Xiwei Sun., "SDUMLA-HMT, A Multimodal Biometric Database", Springer-Verlag Berlin Heidelberg, LNCS 7098, pp. 260-268, 2011

[15] Sangita Bharkad, Manesh Kokare, "Fingerprint Matching using Discreet Wavelet Packet Transform", 3rd IEEE International Advance Computing Conference (IACC), pp. 1183-1188, 2013

[16] Naresh Babu N. T, Annis Fathima A and V. Vaidehi, "An Efficient Face Recognition System Using DWTICA features", IEEE, International Conference on Digital Image Computing: Techniques and Applications, pp. 146-151, 2011

[17] Santosh P. Shrikhande, Hanumant S. Fadewar, "Personal Identification Using Different Biometrics: A Review", International Journal of Engineering Research \& Technology (IJERT), Vol. 3 Issue 2, pp. 1104-1109, February 2014

[18] Shahid Akbar, Ashfaq Ahmad and Maqsood Hayat, "Identification of Fingerprint Using Discrete Wavelet Transform in Conjunction with Support Vector Machine", International Journal of Computer Science Issues (IJCSI), Vol. 11, Issue 5, No 1, pp. 189-199, September 2014

[19] Nabeel Younus Khan, M.Younus Javed, "Efficient Fingerprint Matching Technique Using Wavelet Based
Features", IEEE International Conference on Digital Image Computing Techniques and Applications, pp. 253259, 2007

[20] Jinfeng Yang, Yihua Shi, "Finger-vein ROI localization and vein ridge enhancement", Pattern Recognition Letters, pp. 1559-1579, May 2012

[21] Sunita Lokhande, Vishram. N. Bapat, "Wavelet Packet Based Iris Texture Analysis for Person Authentication", International Journal of Signal \& Image Processing (SIPIJ), Vol.4, No.2, pp. 91-104, April 2013

[22] Nurhafizah Mahri, Shahrel Azmin Sundi Suandi, and Bakhtiar Affendi Rosdi, "Finger Vein Recognition Algorithm Using Phase Only Correlation", IEEE International Conference on Emerging Techniques and Challenges for Hand-Based Biometrics (ETCHB), pp. 16, August 2010

[23] Rongyang Xiao, Gongping Yang, Yilong Yin, and Lu Yang, "A Novel Matching Strategy for Finger Vein Recognition”, Springer -Verlag Berlin Heidelberg LNCS 7751, pp. 364-371, 2013

[24] Zhi Liu and Shangling Song, "An Embedded Real-Time Finger-Vein Recognition System for Mobile Devices", IEEE Transactions on Consumer Electronics, Vol. 58, No. 2, pp. 522-527, May 2012

[25] Gongping Yang, Xiaoming Xi, and Yilong Yin, "Finger Vein Recognition Based on a Personalized Best Bit Map", Hand-Based Biometrics Sensors and Systems, 12 pp.1738-1757, February 2012 